\title{
Prescribed Performance Tracking using State Quantization for Uncertain Feedback Linearizable Systems *
}

\author{
Lampros N. Bikas*, George A. Rovithakis* \\ * Department of Electrical \& Computer Engineering, Aristotle \\ University of Thessaloniki, Thessaloniki 54124, Greece (e-mails: \\ lnmpikas@ece.auth.gr,robi@eng.auth.gr)
}

\begin{abstract}
This paper addresses the problem of imposing pre-defined performance characteristics (by means of maximum steady-state error and minimum convergence rate) on the output tracking errors for a class of uncertain multi-input multi-output (MIMO) nonlinear system in the presence of state quantization implemented by uniform-hysteretic quantizers. A low-complexity control design that requires reduced system knowledge and utilizes only quantized measurements of the state is proposed. The desired performance is achieved by assuming knowledge of the stepsize of the quantizers involved. Simulation results verify the theoretical findings.
\end{abstract}

Keywords: nonlinear control, performance, quantization, uncertain systems

\section{INTRODUCTION}

Quantized control has attracted growing interest recently owing to its theoretical and practical importance in control systems where a finite-capacity communication channel intervenes between the sensors and the controller, i.e., networked control systems (NCSs). In such cases, the communication channel introduces constraints on the feedback information as state measurements have to be quantized before transmitted to the controller. Additionally, even outside the NCS framework, many engineering systems communicate their state information only in quantized form (e.g., robots). State quantization, however, introduces discontinuities in the closed-loop which lead to difficulties in the control design and the stability analysis as the differentiation of the measured signals is infeasible. Furthermore, the quantization errors introduced in the closed-loop deteriorate the overall performance and may even result in stability loss. Therefore, control systems with state quantization deserve special attention.

Linear controlled systems were studied in Corradini et. al. (2008), Lehmann et. al. (2010), Wakaiki et. al. (2018) establishing ultimate boundedness, while in Sharon et. al. (2012) input-to-state stability (ISS) was achieved with respect to external disturbances. The stabilization of nonlinear systems in the sense of ISS was thoroughly examined in Liberzon (2008), Kamaneva et. al. (2008), Liu et. al. (2019) and Ren et. al. (2019) using dynamic quantization

\footnotetext{
* The research leading to these results has received funding by the EU Horizon 2020 Research and Innovation Programme, under grant agreement No 820767, project CoLLaboratE.

Regarding Lampros N. Bikas: This research is co-financed by Greece and the European Union (European Social Fund-ESF) through the Operational Programme "Human Resources Development, Education and Lifelong" in the context of the project "Strengthening Human Resources Research Potential via Doctorate Research" (MIS5000432 ), implemented by the State Scholarships Foundation (IKY)
}

schemes. Further, in Yu et. al. (2011), asymptotic stability is guaranteed by also utilizing dynamic quantization. However, dynamic quantization schemes increase the dynamic order of the system, complicating control design and implementation. Moreover, their extension to tracking problems is currently an open issue.

All aforementioned works assume completely known plant dynamics. In practice, however, uncertainties are almost always encountered. In this direction, parametric uncertainties have been incorporated in De Persis (2009), Vu et. al. (2008), Furtat et. al. (2015), Liu et. al. (2015) and Zheng et. al. (2018), dealing, however, with linear systems. Regarding nonlinear systems with uncertain dynamics, few results have appeared in the literature. In Liu et. al. (2012), unknown nonlinearities have been considered guaranteeing the ISS property of the closed-loop, and recently, in Zhou et. al. (2019), an adaptive backsteppingbased control algorithm was developed to achieve global ultimate boundedness of the system using static, bounded quantizers.

Efforts towards addressing transient and steady-state performance for uncertain nonlinear and quantized systems appeared very recently in Bikas et. al. (2019a), where only control input quantization was considered and in Bikas et. al (2019b), where state quantization was addressed for uncertain single-input single output SISO systems in Brunovsky canonical form. In this work, we extend the aforementioned results to the class of MIMO feedback linearizable, state-quantized systems. The proposed control design is endowed with a low-complexity feature and requires reduced system knowledge. 


\section{PROBLEM FORMULATION}

Consider the MIMO nonlinear system comprised of $m$ subsystems of $n$th order ${ }^{1}$, of the following form; for $i=$ $1, \ldots, m$ and $j=1, \ldots, n-1$,

$$
\begin{aligned}
& \dot{x}_{i, j}=x_{i, j+1}, \\
& \dot{x}_{i, n}=f_{i}(\bar{x}, t)+\sum_{j=1}^{m} g_{i, j}(\bar{x}, t) u_{j}+d_{i}(t),
\end{aligned}
$$

where $\bar{x}=\left[\bar{x}_{1}^{T}, \ldots, \bar{x}_{m}^{T}\right]^{T} \in \mathbb{R}^{m n}$ is the state vector with $\bar{x}_{i}=\left[x_{i, 1}, \ldots, x_{i, n}\right]^{T} \in \mathbb{R}^{n}, i=1, \ldots, m$, and initial condition $\bar{x}(0)=\bar{x}^{0}=\left[x_{i, 1}^{0}, \ldots, x_{i, n}^{0}, \ldots, x_{m, 1}^{0}, \ldots, x_{m, n}^{0}\right]^{T}$. Further, $u=\left[u_{1}, \ldots, u_{m}\right]^{T} \in \mathbb{R}^{m}$ is the control input, $y=\left[x_{1,1}, \ldots, x_{m, 1}\right]^{T} \in \mathbb{R}^{m}$ is the system output, $d_{i}:$ $\mathbb{R}_{\geq 0} \rightarrow \mathbb{R}, i=1, \ldots, m$, are non-measurable disturbance signals and $f_{i}, g_{i, j}: \mathbb{R}^{m n} \rightarrow \mathbb{R}, i, j=1, \ldots, m$, are nonlinear functions. Moreover, consider some desired tracking trajectories $x_{d i}(t): \mathbb{R}_{\geq 0} \rightarrow \mathbb{R}, i=1, \ldots, m$. To proceed, the following assumptions are made:

Assumption 1. The $f_{i}, g_{i, j}$-functions, $i, j=1, \ldots, m$, are locally Lipschitz in $\bar{x}$ and piecewise-continuous in $t$ with unknown analytical expressions.

Assumption 2. The matrix $G_{s}(\bar{x}) \triangleq\left(G(\bar{x})+G^{T}(\bar{x})\right) / 2$ with $G(\bar{x})=\left[g_{i, j}(\bar{x}, t)\right] \in \mathbb{R}^{m \times m}$, denotes the symmetric part of $G$ and is sign definite and its sign denoted by $\operatorname{sgn}\left(G_{s}\right)$ in known.

Assumption 3. There exist unknown constants $\bar{d}_{i} \geq 0$, $i=1, \ldots, m$, satisfying

$$
\left|d_{i}(t)\right| \leq \bar{d}_{i}, i=1, \ldots, m, \forall t \geq 0 .
$$

Assumption 4. The desired tracking trajectories and their derivatives up to $n-1$ are known and satisfy $x_{d i}(t), x_{d i}^{(1)}(t), \ldots, x_{d i}^{(n-1)}(t) \in \mathcal{L}_{\infty}, i=1, \ldots, m$.

Remark 1. Assumption 1 implies that the control solution requires reduced system knowledge as the $f_{i}, g_{i, j}$ functions $i, j=1, \ldots, m$, as well as any corresponding bounds or functions bounding them, are considered unknown. Assumption 2 is common in the literature of strict-feedback systems Krstic et. al. (1995) and imposes a global controllability condition on (1) and Assumptions 3,4 imply the boundedness of the disturbance signals $d_{i}(t)$, the desired trajectories $x_{d i}(t)$, and their derivatives $x_{d i}^{(1)}(t), \ldots, x_{d i}^{(n-1)}(t), i=1, \ldots, m$.

In this work, the state vector $\bar{x}$ immediately after being measured and prior transmitted to the controller. is quantized by uniform-hysteretic quantizers Ceragioli et. al. (2011). Specifically, each coordinate $x_{i, j}, i=1, \ldots, m, i=$ $j, \ldots, n$, of $\bar{x}$ is associated with a quantizer denoted with $q_{i, j}: \mathbb{R} \rightarrow \mathcal{Q}_{i, j}$, where $\mathcal{Q}_{i, j}=\left\{0, \pm \bar{\delta}_{i, j}, \pm 2 \bar{\delta}_{i, j}, 3 \bar{\delta}_{i, j}, \ldots\right\}$. The parameter $\bar{\delta}_{i, j}>0$ represents the step-size of the respective quantizer. It is known Ceragioli et. al. (2011) that each $q_{i, j}$ with step-size $\bar{\delta}_{i, j}>0$, can be decomposed into a linear and a nonlinear part as follows; for $i=1, \ldots, m$, $j=1, \ldots, n$,

$$
q\left(x_{i, j}\right)=x_{i, j}+\delta_{i, j}\left(x_{i, j}\right),\left|\delta_{i, j}\left(x_{i, j}\right)\right| \leq \bar{\delta}_{i, j} .
$$

1 For clarity of presentation, we assumed that all subsystems have identical orders.
In this spirit, we define the measurable error signals for $i=1, \ldots, m, j=0, \ldots, n-1$,

$$
e_{q, i}^{(n-1-j)}(t)=q_{i, j}\left(x_{i, 1}^{(n-1-j)}(t)\right)-x_{d i}^{(n-1-j)}(t) .
$$

The problem addressed in this work reads as follows:

Control Problem: Design a low-complexity, quantized state-feedback controller to meet the following control objectives:

(i) all closed-loop signals remain bounded for all $t \geq 0$,

(ii) the output tracking errors

$$
e_{i}(t)=x_{i, 1}(t)-x_{d i}(t), i=1, \ldots, m,
$$

converge to some residual sets denoted by $\mathcal{E}_{i}=\left\{e_{i} \in\right.$ $\left.\mathbb{R}:\left|e_{i}\right|<\rho_{i}^{\infty}\right\}$ with minimum convergence rate as obtained by the exponential $e^{-\lambda_{i} t}$, where $\lambda_{i} \geq 0$, $\rho_{i}^{\infty}>0$ are pre-defined constants.

Remark 2. In this work, the low-complexity requirement introduces qualitative constraints/characteristics in the controller design. Specifically, no a priori knowledge regarding the controlled system nonlinearities should be employed and no approximation structures (e.g., neural networks, fuzzy systems) should be used to acquire such knowledge. Additionally, no hard calculations (analytic or numerical) should be required to produce the controller output. Finally, the controller should be static to avoid increasing the dynamic order of the closed-loop.

\section{MAIN RESULTS}

In this section the design steps which hold for all $i=$ $1, \ldots, m$ and $t \geq 0$, to solve the Control Problem stated in Section 2 are summarized in the following theorem whose proof is provided in Section 4.

Theorem 1. Consider the MIMO nonlinear system (1) satisfying Assumptions 1-3. Consider also the desired tracking trajectories $x_{d i}(t)$ obeying Assumption 4. Define $T:(-1,1) \rightarrow \mathbb{R}$, a strictly increasing function with locally Lipschitz first order derivative of the following form:

$$
T(\star)=\ln \left(\frac{1+\star}{1-\star}\right) .
$$

For $i=1, \ldots, m$, let

$$
\begin{aligned}
\zeta_{i}^{q}\left(\bar{x}_{i}(t), t\right) & =\sum_{j=0}^{n-1} \frac{(n-1) !}{(n-1-j) ! j !} \kappa_{i}^{j} e_{q, i}^{(n-1-j)}(t), \\
\Delta_{i} & =\sum_{j=0}^{n-1} \frac{(n-1) !}{(n-1-j) ! j !} \kappa_{i}^{j} \bar{\delta}_{i, n-j} .
\end{aligned}
$$

Select the functions

$$
\rho_{i}(t)=\rho_{i}^{0} e^{-\lambda_{i} t}+\kappa_{i}^{n-1} \rho_{i}^{\infty},
$$

with parameters satisfying

$$
\begin{aligned}
\kappa_{i} & >\lambda_{i} \geq 0, \\
\rho_{i}^{\infty} & >\frac{\Delta_{i}}{\kappa_{i}^{n-1}}, \\
\rho_{i}^{0} & >\left|\zeta_{i}^{q}\left(\bar{x}_{i}^{0}\right)\right|+\Delta_{i}-\kappa_{i}^{n-1} \rho_{i}^{\infty} .
\end{aligned}
$$

Let

$$
s_{i}^{q}\left(\zeta_{i}^{q}, t\right)=T\left(\frac{\zeta_{i}^{q}}{\rho_{i}(t)-\Delta_{i}}\right) \in \mathbb{R},
$$


and define

$$
\begin{aligned}
\bar{s}^{q} & =\left[s_{1}^{q}, \ldots, s_{m}^{q}\right]^{T} \in \mathbb{R}^{m}, \\
r_{i}^{q}\left(s_{i}^{q}, t\right) & =\left(\rho_{i}(t)-\Delta_{i}\right)\left(T^{-1}\right)^{(1)}\left(s_{i}^{q}\right) \in \mathbb{R}, \\
R_{q} & =\operatorname{diag}\left(r_{1}^{q}, \ldots, r_{m}^{q}\right) \in \mathbb{R}^{m \times m} .
\end{aligned}
$$

Select some arbitrarily small constants $\epsilon_{i}>0$, design a positive definite matrix $\Gamma=\operatorname{diag}\left(\gamma_{1}, \ldots, \gamma_{m}\right) \in \mathbb{R}^{m \times m}$ such that

$$
\sum_{i=1}^{m}\left(T\left(\frac{\left|\zeta_{i}^{q}(0)\right|+\Delta_{i}+\epsilon_{i}}{\rho_{i}(0)-\Delta_{i}}\right)\right)^{2} \gamma_{i}<1,
$$

and define

$$
w^{q}=\sum_{i=1}^{m}\left(s_{i}^{q}\right)^{2} \gamma_{i} .
$$

Select an arbitrary control gain $k>0$, and design the control input to the system as:

$$
u=-\frac{\operatorname{sgn}\left(G_{s}\right) k}{1-w^{q}} R_{q}^{-1} \Gamma \bar{s}^{q} .
$$

The controller (4)-(14) guarantees:

(i) all signals in the closed-loop are bounded,

(ii) $e_{i}(t)$ converges to $\mathcal{E}_{i}=\left\{e_{i} \in \mathbb{R}:\left|e_{i}(t)\right|<\rho_{i}^{\infty}\right\}$ with minimum convergence rate $e^{-\lambda_{i} t}$.

Remark 3. In (8), the positive constants $\rho_{i}^{\infty}, \lambda_{i}, i=$ $1, \ldots, m$, are selected in view of $(9 \mathrm{a}),(9 \mathrm{~b})$ and pre-define the desired regions $\mathcal{E}_{i}$ and the corresponding convergence rate. Apparently, if $u$ guarantees that $-\rho_{i}^{\star}(t)<e_{i}(t)<$ $\rho_{i}^{\star}(t)$ for some $\rho_{i}^{\star}(t)=\rho_{i}^{\star 0} e^{-\lambda_{i} t}+\rho_{i}^{\infty}$ with $\rho_{i}^{\star 0}>\left|e_{i}(0)\right|$, for all $i=1, \ldots, m$ and for all $t \geq 0$, the proposed controller (4)-(14) provides a solution to the Control Problem stated in Section 2.

Remark 4. The restriction imposed on the selection of $\rho_{i}^{\infty}$ by $(9 \mathrm{~b})$ can be relaxed if the quantizers are considered as design elements. In this case, $(7 \mathrm{~b})$ reveals that is possible to attain smaller values of $\Delta_{i}$ by implementing quantizers with reduced step-size $\bar{\delta}_{i, j}, i=1, \ldots, m, j=1, \ldots, n$.

Remark 5. Notice that the derived control signal $u$ in (14) is well-defined for $w^{q} \neq 1$. In this direction, it will be proven in Section 4 that $w^{q}$ evolves within $\left[0, w^{q *}\right]$ for some $w^{q *}<1$, for all $t \geq 0$, thus evading potential controller singularities.

Remark 6. The controller (4)-(14) is of low-complexity as it satisfies all design requirements of Remark 2.

\section{PROOF OF THEOREM 1}

Define the following compact form of (1):

$$
\dot{\bar{x}}=H(\bar{x}, u, t), \bar{x}(0)=\bar{x}^{0},
$$

where $H(\bar{x}, u, t)=\left[h_{1}^{T}(\bar{x}, u, t), \ldots, h_{m}^{T}(\bar{x}, u, t)\right]^{T} \in \mathbb{R}^{m n}$ and $h_{i}(\bar{x}, u, t)=\left[x_{i, 2}, \ldots, f_{i}(\bar{x}, t)+\sum_{j=1}^{m} g_{i, j}(\bar{x}, t) u_{j}+\right.$ $\left.d_{i}(t)\right]^{T} \in \mathbb{R}^{n}, i=1, \ldots, m$. Further, for stability analysis purposes, let us define the continuously differentiable signals for $i=1, \ldots, m$,

$$
\begin{aligned}
s_{i}^{\min }(t) & =T\left(\frac{\zeta_{i}-\Delta_{i}}{\rho_{i}(t)-\Delta_{i}}\right), \\
s_{i}^{\max }(t) & =T\left(\frac{\zeta_{i}+\Delta_{i}}{\rho_{i}(t)-\Delta_{i}}\right), \\
w(t) & =\sum_{i=1}^{m}\left(s_{i}^{\nu_{i}}(t)\right)^{2} \gamma_{i},
\end{aligned}
$$

where

$$
\begin{aligned}
\zeta_{i}\left(\bar{x}_{i}(t), t\right) & =\sum_{j=0}^{n-1} \frac{(n-1) !}{(n-1-j) ! j !} \kappa_{i}^{j} e_{i}^{(n-1-j)}(t), \\
\nu_{i} & =\left\{\begin{array}{l}
\text { 'min', if } s_{i}<0, \\
\text { 'max', if } s_{i} \geq 0 .
\end{array}\right.
\end{aligned}
$$

Utilizing (3), (4), (10), (16), and the strictly increasing property of the $T$-function, it is not difficult to verify that for all $i=1, \ldots, m$ and $t \geq 0$,

$$
\begin{aligned}
s_{i}^{\min }(t) & \leq s_{i}^{q}(t) \leq s_{i}^{\max }(t), \\
w^{q}(t) & \leq w(t) .
\end{aligned}
$$

By employing (1), (5), (17) and the inverse function derivative formula, differentiation of (16a), (16b) with respect to time yields for $i=1, \ldots, m$, and for all $\alpha \in$ $\{$ 'min','max'\},

$$
\begin{aligned}
\dot{s}_{i}^{\alpha}=\frac{1}{r_{i}\left(s_{i}^{\alpha}\right)} & {\left[f_{i}(\bar{x})+\sum_{j=1}^{m} g_{i, j}(\bar{x}) u_{j}\right.} \\
& \left.+v_{i}\left(\bar{x}_{i}\right)-\dot{\rho}_{i} T^{-1}\left(s_{i}^{\alpha}\right)\right], s_{i}^{\alpha}(0)=s_{i}^{\alpha, 0},
\end{aligned}
$$

where

$$
\begin{aligned}
& v_{i}\left(\bar{x}_{i}\right)=\sum_{j=0}^{n-2} \frac{(n-1) ! \kappa_{i}^{n-1-j}}{(n-1-j) ! j !} e_{i}^{(j+1)}-x_{d i}^{(n)}+d_{i}, \\
& r_{i}\left(s_{i}^{\alpha}\right)=\left(\rho_{i}-\Delta_{i}\right)\left(T^{-1}\right)^{(1)}\left(s_{i}^{\alpha}\right) .
\end{aligned}
$$

The $s_{i}^{\alpha}$-coordinate system (19)-(20), can be written in compact form as

$$
\dot{\bar{s}}=S(\bar{s}, u, t), \bar{s}^{0}=\left[s_{1}^{\alpha, 0}, \ldots, s_{m}^{\alpha, 0}\right]^{T},
$$

where

$$
\begin{aligned}
S & =R^{-1}(\bar{s})\left[F(\bar{x})+G(\bar{x}) u+V(\bar{x})-\dot{P} T^{-1}(\bar{s})\right], \\
\bar{s} & =\left[s_{1}^{\alpha}, \ldots, s_{m}^{\alpha}\right]^{T} \in \mathbb{R}^{m}, \\
R(\bar{s}) & =\operatorname{diag}\left(r_{1}\left(s_{1}^{\alpha}\right), \ldots, r_{m}\left(s_{m}^{\alpha}\right)\right) \in \mathbb{R}^{m \times m}, \\
F(\bar{x}) & =\left[f_{1}(\bar{x}), \ldots, f_{m}(\bar{x})\right]^{T} \in \mathbb{R}^{m}, \\
V(\bar{x}) & =\left[v_{1}\left(\bar{x}_{1}\right), \ldots, v_{m}\left(\bar{x}_{m}\right)\right]^{T} \in \mathbb{R}^{m}, \\
\dot{P} & =\operatorname{diag}\left(\dot{\rho}_{1}, \ldots, \dot{\rho}_{m}\right) \in \mathbb{R}^{m \times m}, \\
T^{-1}(\bar{s}) & =\left[T^{-1}\left(s_{1}^{\alpha}\right), \ldots, T^{-1}\left(s_{m}^{\alpha}\right)\right]^{T} \in \mathbb{R}^{m} .
\end{aligned}
$$

Further, differentiating (16c) with respect to time in view of (16a) and (16b), we obtain the $w$-coordinate system as

$$
\dot{w}=W\left(\bar{s}^{\nu}, u, t\right), w(0)=w^{0},
$$

where

$$
\begin{aligned}
& \bar{s}^{\nu}=\left[s_{1}^{\nu_{1}}, \ldots, s_{m}^{\nu_{m}}\right]^{T} \\
& W=2 \sum_{i=1}^{m}\left[\frac { \gamma _ { i } s _ { i } ^ { \nu _ { i } } } { r _ { i } ( s _ { i } ^ { \nu _ { i } } ) } \left(f_{i}(\bar{x})+\sum_{j=1}^{m} g_{i, j}(\bar{x}) u_{j}\right.\right. \\
&\left.\left.\quad+v_{i}(\bar{x})-\dot{\rho}_{i} T^{-1}\left(s_{i}^{\nu_{i}}\right)\right)\right] .
\end{aligned}
$$


The initial value problem comprised of $(15),(21),(22)$ will be denoted by $\left(\Sigma_{\phi}\right)$ and it is written in compact form as

$$
\dot{\phi}=\sigma(\phi, t), \phi^{0}=\left[\left(\bar{x}^{0}\right)^{T}\left(\bar{s}^{0}\right)^{T} w^{0}\right]^{T},
$$

where $\phi=\left[\bar{x}^{T} \bar{s}^{T} w\right]^{T}, \sigma: \Omega_{\phi} \times[0,+\infty) \rightarrow \mathbb{R}^{n m+m+1}$ with $\Omega_{\phi}=\mathbb{R}^{n m} \times \mathbb{R}^{m} \times\left[0, \omega_{\phi}\right)$, for some constant $\omega_{\phi}>1$, and $\sigma(\phi, t)=\left[\begin{array}{ll}H & S W\end{array}\right]^{T}$.

Recalling that the $T$-function is defined only in $(-1,1), \bar{s}^{0}$ is well defined if and only if $-\rho_{i}(0)+\Delta_{i}<\zeta_{i}^{q}\left(\bar{x}_{i}^{0}\right)<\rho_{i}(0)-$ $\Delta_{i}, i=1, \ldots, m$, which can be easily verified owing to $(3)$, (9b), (9c), (10), (16a) and (16b). Moreover, notice that (12), (13) and (18b) guarantee that $w^{0}<1$; thus by (12) it is further concluded that $w^{0} \in[0,1) \subset\left[0, \omega_{\phi}\right)$. The aforementioned analysis establishes that $\phi^{0} \in \Omega_{\phi}$. Moreover, (24) admits discontinuous right-hand sides owing to the piecewise-continuous control signal $u$. However, following identical arguments as in (Bikas et. al., 2019a, Lemma A.1) and recalling standards theorems Sontag (1995), it is guaranteed that $\left(\Sigma_{\phi}\right)$ has a unique solution

$$
\phi(t) \in \Omega_{\phi}, \forall t \in\left[0, \tau_{f}\right),
$$

in the maximal time interval $\left[0, \tau_{f}\right)$, with $\tau_{f} \in(0,+\infty]$.

The above result, together with (3), (4), (7), (17), (18a) and the properties of the $T$-function, further results in $-\rho_{i}(t)<\zeta_{i}(t)<\rho_{i}(t), i=1, \ldots, m$, for all $t \in\left[0, \tau_{f}\right)$. The latter and the boundedness by construction of $\rho_{i}(t)$, $i=1, \ldots, m$, implies that $\zeta_{i}(t), i=1, \ldots, m$, remains also bounded for all $t \in\left[0, \tau_{f}\right)$. Further, note that (17) is stable with $\zeta_{i}$ acting as the input. Therefore, $e_{i}(t)$ and its derivatives up to $n-1$ remain also bounded for all $t \in\left[0, \tau_{f}\right)$, i.e., there exist constants $e_{i, j}^{\star} \geq 0$ satisfying for $i=1, \ldots, m, j=0, \ldots, n-1$

$$
\left|e_{i}^{(n-1-j)}(t)\right| \leq e_{i, j}^{\star}, \forall t \in\left[0, \tau_{f}\right) .
$$

By (25), (5) and Assumption 4, the existence of a constant $x^{\star}>0$ such that

$$
\|\bar{x}(t)\| \leq x^{\star}, \forall t \in\left[0, \tau_{f}\right)
$$

is guaranteed. Thus, $\bar{x}(t)$ remains within a compact subset of $\mathbb{R}^{n m}$ for all $t \in\left[0, \tau_{f}\right)$. Further, in view of (16) and (17), it is obtained that there exist constants $s_{i}^{\star}>0$ for $i=1, \ldots, m$, such that

$$
\left|s_{i}(t)\right|<s_{i}^{\star}, \forall t \in\left[0, \tau_{f}\right),
$$

which owing to (16a), (16b) and (27), guarantees the existence of constant $c_{w}^{\star}>0$ satisfying

$$
0 \leq w(t)<c_{w}^{\star}, \forall t \in\left[0, \tau_{f}\right) .
$$

Thus, $\bar{s}(t)$ remains within a compact subset of $\mathbb{R}^{m}$ and $w(t)$ evolves strictly within a compact subset of $\mathbb{R}$, for all $t \in\left[0, \tau_{f}\right)$.

To guarantee the implementability of (14), we should establish the existence of a constant $w^{q *}$ such that $0 \leq$ $w^{q}(t) \leq w^{q *}<1$ for all $t \in\left[0, \tau_{f}\right)$. In this direction, define the positive definite and radially unbounded function $V_{L}$ : $\left[0, \tau_{f}\right) \rightarrow \mathbb{R}$ as follows:

$$
V_{L}(t)=\frac{1}{4} w^{2}(t)
$$

Further, let us define $\eta \in\{A, B\}$ and

$$
\begin{aligned}
\bar{s}_{\eta} & =\left[s_{\eta, 1}, \ldots, s_{\eta, m}\right]^{T} \in \mathbb{R}^{m}, \\
R_{\eta} & =\operatorname{diag}\left(r_{1}\left(s_{\eta, 1}\right), \ldots, r_{m}\left(s_{\eta, m}\right)\right) \in \mathbb{R}^{m \times m}, \\
T_{\eta}^{-1} & =\left[T_{\eta}^{-1}\left(s_{\eta, 1}\right), \ldots, T_{\eta}^{-1}\left(s_{\eta, m}\right)\right]^{T} \in \mathbb{R}^{m}, \\
\bar{s}_{N} & =\bar{s}_{A}+\bar{s}_{B}, R_{N}=R_{A}+R_{B}, T_{N}^{-1}=T_{A}^{-1}+T_{B}^{-1},
\end{aligned}
$$

where

$$
\begin{aligned}
& s_{A, i}=s_{i}^{\nu_{i}}, s_{B, i}=0, \text { if } \operatorname{sgn}\left(s_{i}^{\min }\right)=\operatorname{sgn}\left(s_{i}^{\max }\right), \\
& s_{A, i}=0, s_{B, i}=s_{i}^{\nu_{i}}, \text { if } \operatorname{sgn}\left(s_{i}^{\min }\right) \neq \operatorname{sgn}\left(s_{i}^{\max }\right) .
\end{aligned}
$$

Utilizing the aforementioned definitions and differentiating $V_{L}$ with respect to time yields for all $t \in\left[0, \tau_{f}\right)$,

$$
\begin{gathered}
\dot{V}_{L} \stackrel{(22)}{\leq} w\left(\|V\|+\|F\|+\left\|\dot{P} T_{N}^{-1}\right\|\right)\left\|R_{N}^{-1} \Gamma \bar{s}_{N}\right\| \\
+w u^{T} G^{T} R_{A}^{-1} \Gamma \bar{s}_{A}+w u^{T} G^{T} R_{B}^{-1} \Gamma \bar{s}_{B} .
\end{gathered}
$$

To proceed, note that by the continuity of $T^{-1}(\cdot),(27)$, and the Extreme Value Theorem, the existence of constants $\underline{\tau}_{i}^{*}, \bar{\tau}_{i}^{*}>0$ such that $0<\underline{\tau}_{i}^{*} \leq\left(T^{-1}\right)^{(1)}(\cdot) \leq \bar{\tau}_{i}^{*}$ for all $t \in\left[0, \tau_{f}\right)$, and $i=1, \ldots, m$, is concluded. Utilizing the latter, $(20 \mathrm{~b})$, and the boundedness by construction of $\rho_{i}, i=1, \ldots, m$, it is further concluded that there exist constants $\underline{r}_{i}^{*}, \bar{r}_{i}^{*}>0$ such that

$$
0<\underline{r}_{i}^{*} \leq r_{i}(\cdot) \leq \bar{r}_{i}^{*}, i=1, \ldots, m, \forall t \in\left[0, \tau_{f}\right) .
$$

Recalling further Assumptions 1, 3, 4, (20a) and (25), it is concluded that there exist constants $\theta_{1}^{\star}, \theta_{2}^{\star} \geq 0$ such that for all $t \in\left[0, \tau_{f}\right)$,

$$
\begin{aligned}
\|V\|+\|F\|+\left\|\dot{P} T_{N}^{-1}\right\| & \leq \theta_{1}^{\star}, \\
\left\|R_{N}^{-1} \Gamma \bar{s}_{N}\right\| & \leq \theta_{2}^{\star} .
\end{aligned}
$$

Hence, $\dot{V}_{L}$ becomes for all $t \in\left[0, \tau_{f}\right)$,

$$
\begin{array}{r}
\dot{V}_{L} \leq w\left(\theta_{1}^{\star} \theta_{2}^{\star}+u^{T} G^{T} R_{A}^{-1} \Gamma \bar{s}_{A}+u^{T} G^{T} R_{B}^{-1} \Gamma \bar{s}_{B}\right) \\
\stackrel{(14)}{=} w\left(\theta_{1}^{\star} \theta_{2}^{\star}-\frac{\operatorname{sgn}\left(G_{s}\right) k}{1-w^{q}}\left(R_{q}^{-1} \Gamma \bar{s}^{q}\right)^{T} G^{T} R_{A}^{-1} \Gamma \bar{s}_{A}\right. \\
\left.-\frac{\operatorname{sgn}\left(G_{s}\right) k}{1-w^{q}}\left(R_{q}^{-1} \Gamma \bar{s}^{q}\right)^{T} G^{T} R_{B}^{-1} \Gamma \bar{s}_{B}\right) .
\end{array}
$$

To proceed, the following result, whose proof can be found in the Appendix, is required:

Proposition 1. For all $i=1, \ldots, m$ and $t \in\left[0, \tau_{f}\right)$,

(a) If $\operatorname{sgn}\left(s_{i}^{\min }\right)=\operatorname{sgn}\left(s_{i}^{\max }\right)$, there exist constants $\mu_{s i}^{\star}, \mu_{r i}^{\star}>0$ such that $s_{i}^{q}=\mu_{s i}^{\star} s_{i}^{\nu_{i}}$ and $r_{i}^{q}=\mu_{r i}^{\star} r_{i}\left(s_{i}^{\nu_{i}}\right)$.

(b) If $\operatorname{sgn}\left(s_{i}^{\min }\right) \neq \operatorname{sgn}\left(s_{i}^{\max }\right)$, there exist positive constants $\xi_{s q i}^{\star}, \xi_{s i}^{\star}>0$ and $\xi_{w}^{\star} \in(0,1)$, such that $\left|s_{i}^{q}\right| \leq$ $\xi_{s q i}^{\star},\left|s_{i}^{\nu_{i}}\right| \leq \xi_{s i}^{\star}$ and $w^{q}<\xi_{w}^{\star}$.

(c) There exist constant $\mu_{w}^{\star} \in(0,1)$ such that $w^{q}=\mu_{w}^{\star} w$.

Further, by the continuity of $g_{i, j}, i, j=1, \ldots, m$, it is concluded via the Extreme Value Theorem the existence of constants $\bar{g}_{i, j}>0$ such that $\left|g_{i, j}(\cdot)\right| \leq \bar{g}_{i, j}$ for all $t \in\left[0, \tau_{f}\right)$. By applying the aforementioned result, Proposition 1 and (29), $\dot{V}_{L}$ becomes for all $t \in\left[0, \tau_{f}\right)$,

$$
\dot{V}_{L} \leq w\left[\theta^{\star}-\frac{\operatorname{sgn}\left(G_{s}\right) k}{1-\mu_{w}^{\star} w}\left(R_{A}^{-1} \Gamma \bar{s}_{A}\right)^{T} M G^{T} R_{A}^{-1} \Gamma \bar{s}_{A}\right],
$$

where 


$$
\begin{aligned}
\theta^{\star} & =\theta_{1}^{\star} \theta_{2}^{\star}+\frac{k}{1-\xi_{w}^{\star}} \sum_{i \in \mathcal{B}}\left(\frac{\gamma_{i}^{2} \xi_{s q i}^{\star} \xi_{s i}^{\star}}{\left(\underline{r}_{i}^{\star}\right)^{2}} \sum_{j=1}^{m} \bar{g}_{i, j}\right)>0, \\
\mathcal{B} & =\left\{i \in\{1, \ldots, m\}: s_{B, i} \neq 0\right\} \\
M & =\operatorname{diag}\left(M_{1}, \ldots, M_{m}\right) \in \mathbb{R}^{m \times m}, \\
M_{i} & =\left\{\begin{array}{l}
\frac{\mu_{s i}^{\star}}{\mu_{r i}^{\star}}, \text { if } \operatorname{sgn}\left(s_{i}^{\min }\right)=\operatorname{sgn}\left(s_{i}^{\max }\right), \\
0, \text { if } \operatorname{sgn}\left(s_{i}^{\min }\right) \neq \operatorname{sgn}\left(s_{i}^{\max }\right) .
\end{array}\right.
\end{aligned}
$$

Moreover, by utilizing the positive definiteness of $M$, and adopting the line of proof in Theodorakopoulos et. al. (2016), the existence of constants $g_{\phi}^{\star}, h_{\phi}^{\star}>0$ satisfying for all $t \in\left[0, \tau_{f}\right)$,

$$
\begin{gathered}
-\operatorname{sgn}\left(G_{s}\right)\left(R_{A}^{-1} \Gamma \bar{s}_{A}\right)^{T} M G^{T} R_{A}^{-1} \Gamma \bar{s}_{A} \leq-g_{\phi}^{\star}\left\|R_{A}^{-1} \Gamma \bar{s}_{A}\right\|^{2}, \\
\left\|R_{A}^{-1} \Gamma \bar{s}_{A}\right\|^{2} \geq h_{\phi}^{\star} w,
\end{gathered}
$$

Utilizing the latter, $\dot{V}_{L}$ becomes for all $t \in\left[0, \tau_{f}\right)$,

$$
\dot{V}_{L} \leq \frac{w}{1-\mu_{w}^{\star} w}\left(\theta^{\star}-\left(\theta^{\star} \mu_{w}^{\star}+g_{\phi}^{\star} h_{\phi}^{\star} k\right) w\right),
$$

which yields $\dot{V}_{L}(t)<0$, for all $t \in \mathcal{T}_{\phi}$, where $\mathcal{T}_{\phi}=$ $\left\{t \in\left[0, \tau_{f}\right): w>w^{\star}\right\}$, with $w^{\star}=\theta^{\star} /\left(\theta^{\star} \mu_{w}^{\star}+g_{\phi}^{\star} h_{\phi}^{\star} k\right)$. Consequently,

$$
0 \leq w \leq \bar{w}, \forall t \in\left[0, \tau_{f}\right),
$$

where $\bar{w}=\max \left\{w^{\star}, w^{0}\right\}$, with $w^{0}<1$. Utilizing (12), (31), and Proposition 1(c), we conclud the existence of a constant $w^{q *} \in(0,1)$ such that

$$
0 \leq w^{q} \leq w^{q *}<1,
$$

which further implies that the control solution (14) is welldefined for all $t \in\left[0, \tau_{f}\right)$. Finally, by (26), (27) and (31), it is concluded that the solution $\phi(t)$ of $\left(\Sigma_{\phi}\right)$ evolves within a compact subset of $\Omega_{\phi}$ for all $t \in\left[0, \tau_{f}\right)$. Therefore, following standard arguments (Khalil, 2001, Theorem 3.3), the solution is extended to $\tau_{f}=+\infty$.

The above result, and (16a), (16b) further guarantees that for all $i=1, \ldots, m$,

$$
-\rho_{i}(t)<\zeta_{i}(t)<\rho_{i}(t), \forall t \geq 0 .
$$

Hence, by (Theodorakopoulos et. al., 2016, Lemma 2), it is concluded that there exist constants $\rho_{i, j}^{\star 0}>0, i=1, \ldots, m$, $j=0, \ldots, n-2$, such that

$$
\left|e_{i}(t)\right|<\rho_{i, j}^{\star 0} e^{-\lambda_{i} t}+\rho_{i}^{\infty}, \forall t \geq 0,
$$

which further implies that the output tracking errors $e_{i}(t)$ converge to $\mathcal{E}_{i}=\left\{e_{i} \in \mathbb{R}:\left|e_{i}\right|<\rho_{i}^{\infty}\right\}$ with minimum convergence rate as obtained by $e^{-\lambda_{i} t}$, thus concluding the Proof of Theorem 1.

\section{SIMULATION RESULTS}

To clarify and verify the theoretical findings, simulation studies were performed on a two-link robotic manipulator Bechlioulis et. al. (2008), whose parameters were selected as $m_{1}=1.5[\mathrm{kgr}], m_{2}=1[\mathrm{kgr}], l_{1}=0.25[\mathrm{~m}], l_{2}=0.2[\mathrm{~m}]$, $I_{1}=0.96\left[\mathrm{kgr} \mathrm{m}{ }^{2}\right], I_{2}=0.81\left[\mathrm{kgr} \mathrm{m}{ }^{2}\right]$ and $g=9.81\left[\mathrm{~m} / \mathrm{s}^{2}\right]$. The system output is $y=\left[\begin{array}{ll}\theta_{1} & \theta_{2}\end{array}\right]^{T}$, where $\theta_{1}, \theta_{2}$ denote the positions of the two links, respectively. The system initially rests at $\theta_{1}(0)=\pi / 14, \theta_{2}(0)=\pi / 16$. The control objective is to drive the output from its initial condition to $(\pi / 10, \pi / 10)$ in $6 \mathrm{~s}$, following the desired polynomial trajectories $\theta_{d 1}(t)=\theta_{d 2}(t)=(\pi / 10)\left(10 t^{3} / 6^{3}-15 t^{4} / 6^{4}+\right.$ $\left.t^{2} / 6\right)$. Both position errors $e_{i}=\theta_{i}-\theta_{d i}, i=1,2$, are
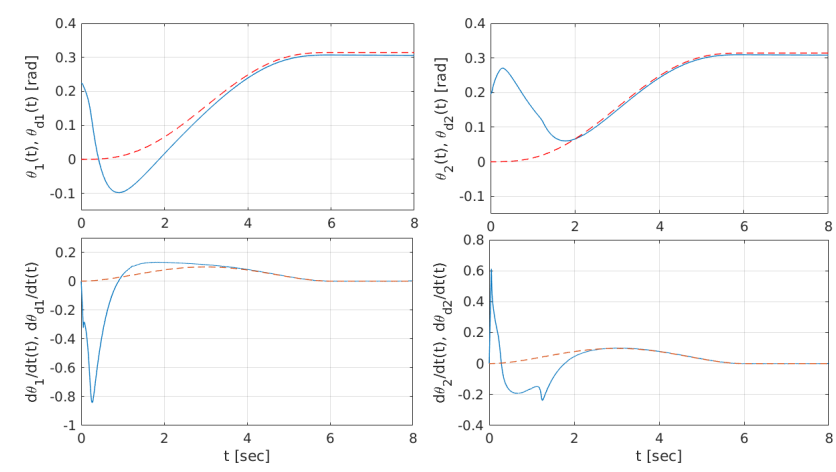

Fig. 1. The states of the system $\theta_{1}, \theta_{2}, \dot{\theta}_{1}, \dot{\theta}_{2}$ (solid lines) together with the desired tracking trajectories $\theta_{d 1}, \theta_{d 2}, \dot{\theta}_{d 1}, \dot{\theta}_{d 2}$ (dashed lines).

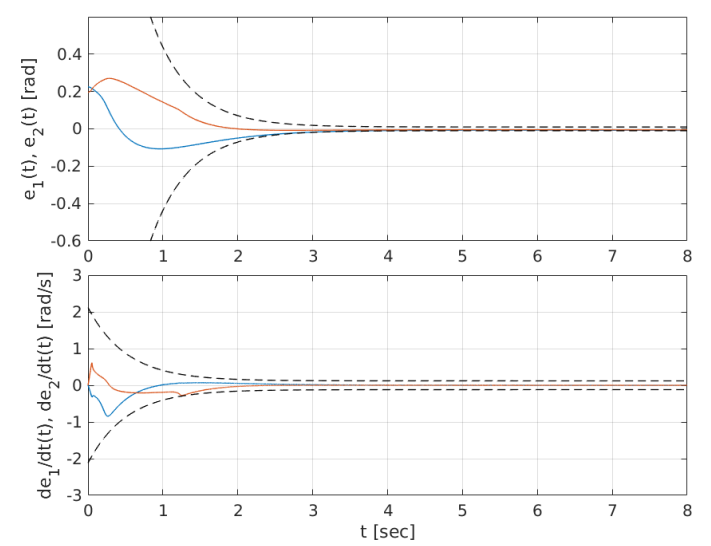

Fig. 2. The output tracking errors $e_{1}, e_{2}$ and the velocity errors $\dot{e}_{1}=\dot{\theta}_{1}-\dot{\theta}_{d 1}, \dot{e}_{2}=\dot{\theta}_{2}-\dot{\theta}_{d 2}$ (solid lines) alongside the corresponding performance bounds (dashed lines).

required to converge to $\mathcal{E}_{i}=\left\{e_{i} \in \mathbb{R}:\left|e_{i}\right|<0.01\right\}$, $i=1,2$, with minimum convergence rate as obtained by the exponential $e^{-1.9 t}$. The link positions and their velocities enter the controller in quantized form. Uniformhysteretic quantizers having step-size 0.002 are utilized for the former measurements and 0.001 for the latter. Further, the disturbance signals are assumed to be: $d_{1}(t)=$ $0.25 \sin (t)$ and $d_{2}(t)=0.75 \cos (t)$.

To achieve the aforementioned control objective we selected $\rho_{1}(t)=\rho_{2}(t)=2.8 e^{-1.9 t}-0.02$ with parameters satisfying (9a)-(9c); $\lambda_{1}=\lambda_{2}=1.9, \kappa_{1}=\kappa_{2}=2$, $\rho_{i}^{\infty}=0.01>\Delta_{i} / \kappa_{i}=0.0025, i=1,2, \rho_{1}^{0}=2.8>\left|\zeta_{1}^{q}(0)\right|+$ $\Delta_{1}-\kappa_{1} \rho_{1}^{\infty}=0.471$ and $\rho_{2}^{0}=2.8>\left|\zeta_{2}^{q}(0)\right|+\Delta_{2}-$ $\kappa_{2} \rho_{2}^{\infty}=0.415$. Further, $\gamma_{1}=\gamma_{2}=0.05, \epsilon_{1}=\epsilon_{2}=0.1$ and $k=220$. Therefore, the control signals are given as follows; for $i=1,2$,

$$
u_{i}=\frac{k \gamma_{i}\left(\rho_{i}(t)-\Delta_{i}\right) s_{i}^{q}}{T^{(1)}\left(s_{i}^{q}\right)\left(\gamma_{1}\left(s_{1}^{q}\right)^{2}+\gamma_{2}\left(s_{2}^{q}\right)^{2}-1\right)}, s_{i}^{q}=\frac{\dot{e}_{q, i}+\kappa_{i} e_{q, i}}{\rho_{i}(t)-\Delta_{i}},
$$

where $e_{q, i}=q_{s, 1}\left(\theta_{i}\right)-\theta_{d i}$ and $\dot{e}_{q, i}=q_{s, 2}\left(\dot{\theta}_{i}\right)-\dot{\theta}_{d i}$.

The results are depicted in Figs. 1-3, clearly verifying the theoretical findings. Specifically, Fig. 1 shows the states of the system together with the desired tracking trajectories. The output tracking errors alongside the corresponding performance bounds are shown in Fig. 2. Finally, Fig. 3 illustrates the the control inputs applied to the system. 


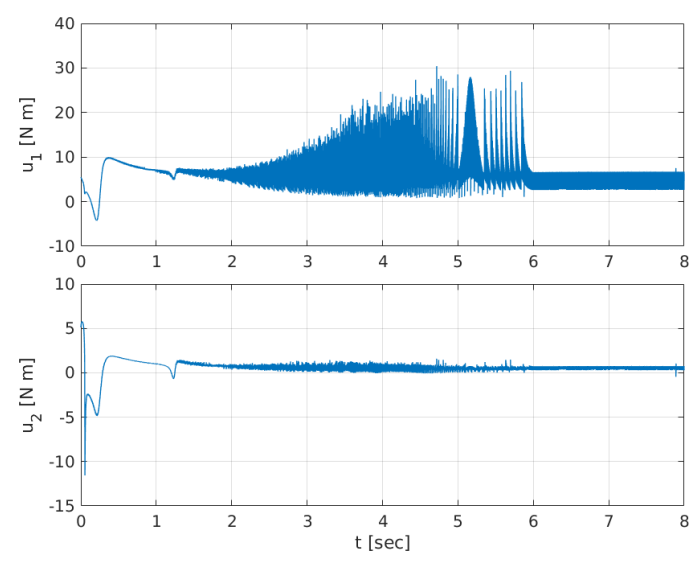

Fig. 3. The requested control inputs.

\section{Appendix A. PROOF OF PROPOSITION 1}

a) By employing (16a), (16b) and (18a), we straightforwardly deduce that $\operatorname{sgn}\left(s_{i}^{q}\right)=\operatorname{sgn}\left(s_{i}^{\min }\right)=\operatorname{sgn}\left(s_{i}^{\max }\right)$ for all $\zeta_{i} \in \mathbb{R}-\left\{\left[-\Delta_{i}, \Delta_{i}\right]\right\}$, which implies the existence of constants $\mu_{s i}^{\star}, \mu_{r i}^{\star}>0$ such that $s_{i}^{q}=\mu_{s i}^{\star} s_{i}^{\nu_{i}}$ and $r_{i}^{q}=\mu_{r i}^{\star} r_{i}\left(s_{i}^{\nu_{i}}\right)$.

b) Following the proof of (a), we obtain that $\operatorname{sgn}\left(s_{i}^{\min }\right) \neq$ $\operatorname{sgn}\left(s_{i}^{\max }\right)$ if and only if $\zeta_{i} \in\left[-\Delta_{i}, \Delta_{i}\right]$. Hence, recalling (16a), (16b) and the boundedness by construction of $\rho_{i}$, we conclude the existence of constants $\xi_{s q i}^{\star}, \xi_{s i}^{\star}>0$ such that $\left|s_{i}^{q}(t)\right| \leq \xi_{s q i}^{\star}$ and $\left|s_{i}^{\nu_{i}}(t)\right| \leq \xi_{s i}^{\star}$ for all $\zeta_{i} \in$ $\left[-\Delta_{i}, \Delta_{i}\right]$. Moreover, owing to (3) and (12), the signals $\hat{w}=\sum_{i=1}^{m}\left(\hat{s}_{i}\right)^{2} \gamma_{i}$ with $\hat{s}_{i}=\left(\zeta_{i}+\Delta_{i}+\xi_{i}\right) /\left(\rho_{i}-\Delta_{i}\right)$, $i=1, \ldots, m$, satisfy $\hat{w}(0)<1$, and therefore, $0 \leq \hat{w}(t)<1$ for all $t \in\left[0, \tau_{f}\right)$. Employing the latter, the definitions of $w^{q}$ and $w$ in (13) and (16) respectively, and (18b), it is not difficult to verify that if $\zeta_{i} \in\left[-\Delta_{i}, \Delta_{i}\right]$, there exist constants $\xi_{w}^{\star}$ such that $w^{q}<\xi_{w}^{\star}$.

c) By utilizing the definitions of $w^{q}$ and $w$ in (13) and (16c) respectively, we straightforwardly conclude that $w^{q}, w \geq 0$. Employing further (18b), we guarantee the existence of a constant $\mu_{w}^{\star} \in(0,1)$.

\section{REFERENCES}

Bechlioulis, C., and Rovithakis, G. (2008). Robust adaptive control of feedback linearizable MIMO nonlinear systems with prescribed performance. IEEE Trans. Autom. Control, vol. 53(9), 2090-2099.

Bikas, L., and Rovithakis, G. (2019a). Combining prescribed tracking performance and controller simplicity for a class of uncertain MIMO nonlinear systems with input quantization. IEEE Trans. Autom. Control, vol. 64(3), 1228-1235.

Bikas, L. and Rovithakis, G. (2019b). Prescribed tacking performance under state-feedback quantization for uncertain nonlinear systems. 2019 18th European Control Conference (ECC), Naples, Italy, 25-28 June.

Ceragioli, F., De Persis, C., and Frasca P. (2011). Discontinuities and hysteresis in quantized average consensus. Automatica, vol. 47, 1916-1928.

Corradini, M. L., and Orlando, G. (2008). Robust quantized feedback stabilization of linear systems. Automatica, vol. 44, 2458-2462.
De Persis, C. (2009). Robust stabilization on nonliear systems with quantized and ternary control. Systems \& Control Letters, vol. 58, 602-608.

Furtat, I. B., Fradkov, A. L., and Liberzon, D. (2015). Compensation of disturbances for MIMO systems with quantized output. Automatica, vol. 60, 239-244.

Kamaneva, T., and Nesic, D. Input-to-state stabilization of nonlinear systems with quantized feedback. 17th World Congress The International Federation of Automatic Control, Seoul, Korea, 6-11 July.

Khalil, H. (2001). Nonlinear Systems. 3rd ed. NJ, USA: Prentice Hall.

Krstic, M., Kanellakopoulos, I., and Kokotovic, P. V. (1995). Nonlinear and adaptive control design. New York, Willey.

Lehmann, D., and Lunze, J. (2010). Event-based control using quantized state information. 2nd IFAC Workshop on Distributed Estimation and Control in Networked Systems, Annecy, France, 13-14 September.

Liberzon, D. (2008). Observer-based quantized output feedback control of nonlinear systems. 17th World Congress The International Federation of Automatic Control, Seoul, Korea, 6-11 July.

Liu, T., Jiang, Z., and Hill, D. J. (2012). A sector bound approach to feedback control of nonlinear systems with state quantization. Automatica, vol. 48, 145-152.

Liu, T., and Jiang, Z. (2019). Event-triggered control of nonlinear systems with state quantization. IEEE Trans. Autom. Control, vol. 64(2), 797-803.

Liu, K., Fridman, E., and Johansson, K. H. (2015). Dunamic quantization of uncertain linear networked control systems. Automatica, vol. 59, 248-255.

Ren, W., and Xiong, J. (2019). Quantized feedback stabilization of nonlinear systems with external disturbance. IEEE Trans. Autom. Control, vol. 63(9), 3167-3172.

Sharon, Y., and Liberzon, D. (2012). Input-to-state stabilizing controller for systems with coarse quantization. IEEE Trans. Autom. Control, vol. 57(4), 830-844.

Sontag, E.D. (1995). Mathematical Control Theory. New York, USA: Springer-Verlag.

$\mathrm{Yu}$, S., and Wang, X. (2011). Quantization scheme design in distributed event-triggered networked control systems. 18th IFAC World Congress, Milano, Italy, 28 August - 2 September.

Theodorakopoulos, A., and Rovithakis, G. (2016). Lowcomplexity prescribed performance control of uncertain MIMO feedback linearizable systems. IEEE Trans. Autom. Control, vol. 61(7), 1946-1952.

$\mathrm{Vu}$, L., and Liberzon, D. (2008). Stabilizaing uncertain systems with dynamic quantization. 47th IEEE Conference on Decision and Control (CDC), Cancun, Mexico, 9-11 December.

Wakaiki, M., and Yamamoto, Y. (2016). Stability analysis of sampled-data switched systems with quantization. Automatica, vol. 69, 157-168.

Zheng, B., Yu, X., and Xue, Y. (2018). Quantized feedback sliding-mode control: an event-triggered approach. Automatica, vol. 91, 126-135.

Zhou, J., Wen, C., Wang, W., and Yang, F. (2019). Adaptive backstepping control of nonlinear uncertain systems with quantized states. IEEE Trans. Autom. Control (Early Access), (DOI:10.1109/TAC.2019.2906931). 